\title{
The Design and Analysis of a Miller-Divider-Based Clock Generator for MBOA-UWB Application
}

\author{
Tai-Cheng Lee, Member, IEEE, and Yen-Chuan Huang
}

\begin{abstract}
A Miller-divider-based clock generator is proposed for Multi-Band OFDM Alliance (MBOA) ultrawideband (UWB) application. Employing closed-loop operation, the clock generator can produce three different carrier frequencies with negligible in-band spurs. The settling time of the proposed clock generator is analyzed based on a linear feedback system. A transistor sizing optimization technique for active inductors with a current-reusing technique is used to achieve low-power operation and area saving. Fabricated in a 0.18- $\mu \mathrm{m}$ technology, the clock generator achieves less than 9.5-ns settling time while dissipating less than $47 \mathrm{~mW}$ from a 1.8-V power supply.
\end{abstract}

Index Terms-Frequency synthesizer, Miller divider, ultrawideband (UWB).

\section{INTRODUCTION}

$\mathbf{U}$ WB is a high-speed and short-range wireless technology, utilizing the unlicensed radio spectrum from $3.1 \mathrm{GHz}$ to $10.6 \mathrm{GHz}$. Mode-1 operation uses the spectrum from $3.1 \mathrm{GHz}$ to $4.8 \mathrm{GHz}$, which is considered to be feasible and practical for the current CMOS technologies. The 1.7-GHz bandwidth is sufficient for three sub-bands of $500 \mathrm{MHz}$, as illustrated in Fig. 1. The center frequencies of the three bands are $3432 \mathrm{MHz}$, $3960 \mathrm{MHz}$, and $4488 \mathrm{MHz}$, respectively. UWB systems require less than 9.5-ns frequency-hopping time to obtain the best performance. Conventional phase-locked loop (PLL)-based frequency synthesizers require about hundreds of input reference cycles to settle. Therefore, if a PLL with input reference about $500 \mathrm{MHz}$ and loop bandwidth is chosen to be $50 \mathrm{MHz}$, the settling time is approximately at the order of tens of microseconds. Thus, it is almost impossible to achieve the fast frequency-hopping time by a PLL-based frequency synthesizer.

Single-sideband (SSB) mixing is an alternative to synthesizing frequencies agilely [1], [2]. Shown in Fig. 2, the output of SSB mixing can be expressed as

$$
\cos \omega_{1} t \cos \omega_{2} t \pm \sin \omega_{1} t \sin \omega_{2} t=\cos \left(\omega_{1} \mp \omega_{2}\right) t .
$$

Equation (1) shows that the SSB mixing architecture generates two output frequencies by controlling the sign of the adder. For MBOA-UWB mode- 1 application, the carrier frequency can be obtained by choosing $3960 \mathrm{MHz}$ for $\omega_{1}$ and $528 \mathrm{MHz}$ or 0 for $\omega_{2}$.

Manuscript received July 29, 2005; revised January 10, 2006. This work was supported by the National Science Council of Taiwan under Contract 93-2220-E-002-010-, and by Mediatek.

The authors are with the Department of Electrical Engineering and Graduate Institute of Electronics Engineering, National Taiwan University, Taipei, Taiwan 106, R.O.C. (e-mail: tlee@cc.ee.ntu.edu.tw).

Digital Object Identifier 10.1109/JSSC.2006.874279

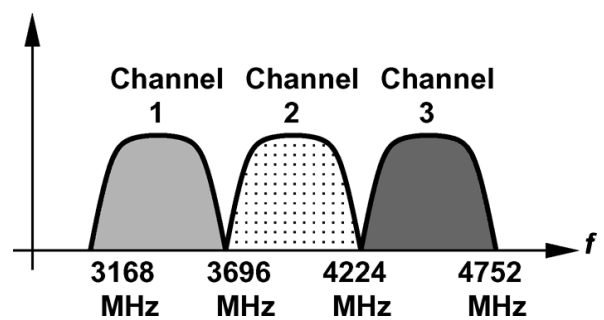

Fig. 1. Frequency plan for mode-1 MBOA-UWB operation.

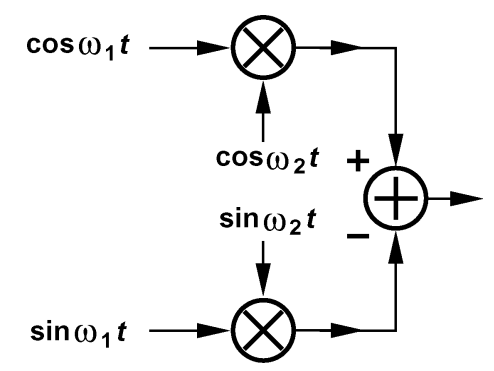

Fig. 2. Ideal single-sideband mixing

The SSB mixing topology requires quadrature clocks of both input frequencies. However, to generate the quadrature signals for SSB mixing requires precise matching of circuits as well as significant power. Furthermore, in the presence of a gain mismatch of $\varepsilon$ and phase imbalance of $\theta$, (1) becomes

$$
\begin{aligned}
v_{\text {out }}(t)= & V_{0} \sin \omega_{1} t \sin \omega_{2} t \\
& +V_{0}(1+\varepsilon) \cos \omega_{1} t \cos \left(\omega_{2} t+\theta\right) \\
\approx & \frac{V_{0}}{2}[1+(1+\varepsilon) \cos \theta] \cos \left(\omega_{1}-\omega_{2}\right) t \\
& -\frac{V_{0}}{2}(1+\varepsilon) \sin \theta \sin \left(\omega_{2}-\omega_{1}\right) t \\
& +\frac{V_{0}}{2}[-1+(1+\varepsilon) \cos \theta] \cos \left(\omega_{1}+\omega_{2}\right) t \\
& -\frac{V_{0}}{2}(1+\varepsilon) \sin \theta \sin \left(\omega_{2}+\omega_{1}\right) t
\end{aligned}
$$

Equation (3) shows that the single-sideband mixing generates an unwanted sideband at $\omega_{1}+\omega_{2}$ and this sideband falls in the other channel. Thus, the in-band spurious tones translate the adjacent channel interferences down to the baseband and corrupt the signal in the desired channel. Furthermore, frequency leakage of $\omega_{1}$ generates another sideband spur. Without any calibration, both carrier leakage and unwanted sideband are typically on the order of $-30 \mathrm{~dB}$. 


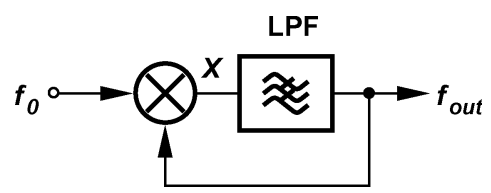

Fig. 3. Regenerative (Miller) divider.

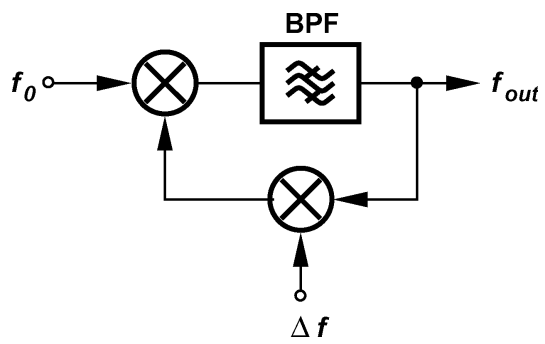

Fig. 4. Modified Miller divider.

In Section II, a closed-loop clock generator is proposed to generate the three carrier frequencies with negligible in-band spurs. This architecture requires only differential signals and generates major spurs which are out of the band of interest. Section III illustrates the major building blocks of this architecture, while Section IV and V outlines the experimental results and the conclusion, respectively.

\section{Clock Generator Architecture}

A closed-loop clock generator based on a regenerative frequency divider is introduced to generate high-precision carrier signal [3]. Spur frequency locations and channel-switching time will be analyzed based on the proposed model.

\section{A. Architecture of the Proposed Clock Generator}

1) Regenerative (Miller) Frequency Divider: Fig. 3 shows a regenerative frequency divider which is originally proposed by Miller [4]. A Miller divider contains only one mixer and a lowpass filter or a bandpass filter. The mixer directly mixes the input signal with the output signal, and the loop filter removes the unwanted frequency component at the output of the mixer. If the mixer is ideal, the signal at node $X$ contains only two frequency components, $f_{0}-f_{\text {out }}$ and $f_{0}+f_{\text {out }}$. Then, the loop filter can provide enough suppression for higher frequency component and the frequency-division mechanism can be expressed as

$$
f_{0}-f_{\text {out }}=f_{\text {out }}, \quad f_{\text {out }}=\frac{f_{0}}{2} .
$$

Equation (4) shows that under proper phase and gain conditions, the component at $f_{0} / 2$ will remain and circulate around the loop [5]. Since the device parasitic capacitances can be merged in the low-pass filter, this topology achieves a high-speed operation and is widely adopted in the design of high-frequency divider.

2) Modified Miller Divider: Shown in Fig. 4, a modified Miller divider can be constructed by inserting a mixer in the feedback path instead of mixing the input signal with the output signal directly. Assuming both mixers are ideal and the fre-

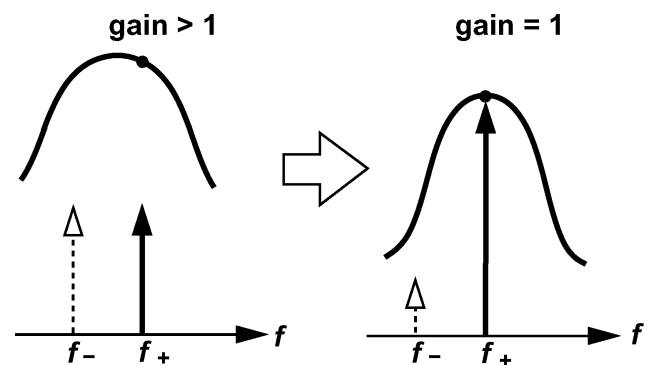

Fig. 5. Loop-gain variation of the two frequency solutions in the modified Miller divider.

quency $\Delta f$ is smaller than the input frequency $f_{0}$, the output signal frequency can be expressed as

$$
f_{0}-\left(f_{\text {out }} \pm \Delta f\right)=f_{\text {out }}, \quad f_{\text {out }}=\frac{f_{0}}{2} \pm \frac{\Delta f}{2} .
$$

Two possible frequencies satisfy (5), but only one unique frequency can exist in the loop. The loop will adjust itself to sustain a unique major frequency signal. Shown in Fig. 5, even if both frequency components have loop gain greater than one, the frequency solution with larger loop gain grows rapidly and its corresponding signal saturates the circuit due to the circuit nonlinearity or other amplitude limiting mechanism in the loop. Therefore, the frequency solution with lower loop gain is suppressed and disappears in the steady state.

The above analysis shows that adjustments in the bandpass filter's center frequency will effectively control the output frequency. If the center frequency is tuned to the higher frequency, the output signal comprises only the higher frequency solution and vice versa. The quality factor $(Q)$ of the bandpass filter needs not to be high as long as the two possible frequency signals can have distinguishable loop gain. Only the higher loop gain frequency will survive even if the difference in the loop gains was initially very small. This property can greatly reduce the design difficulty of a bandpass filter.

Fig. 6 shows the proposed architecture, which can generate the three carrier frequencies for MBOA-UWB mode-1 system. The illustrated Miller-divider-based clock generator contains two feedback loops, however these two loops are not enabled simultaneously. If the direct feedback path is closed, the circuit becomes simply a Miller divider. Otherwise, it is the modified Miller divider. Therefore, by changing the control signals of the switches and the center frequency of the bandpass filter accordingly, three specific frequencies $\left(f_{0}+\Delta f\right) / 2, f_{0} / 2$, and $\left(f_{0}-\Delta f\right) / 2$ are obtained.

For MBOA-UWB mode-1 system, $f_{0}$ is equal to $7.92 \mathrm{GHz}$, two times of the channel- 2 carrier frequency and $\Delta f$ is equal to $1.056 \mathrm{GHz}$, two times of channel spacing. $f_{0}$ can be generated by a PLL-based frequency synthesizer and $\Delta f$ frequency can be derived from $f_{0}$ via a divide-by-7.5 divider, shown in the next section.

\section{B. Analysis of the Modified Miller Divider}

The proposed clock generator exhibits unique frequency selectivity, but nonideal frequency spurs may occur in other fre- 


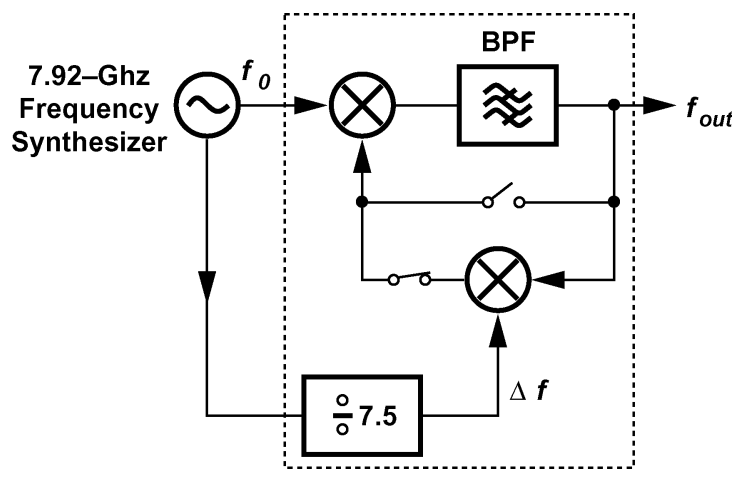

Fig. 6. Combination of Miller divider and modified Miller divider.

quencies. In addition to spur analysis, time-domain analysis is performed to derive the settling time of the proposed clock generators.

1) Spur Analysis: The architecture shown in Fig. 4 has two possible output frequencies but only one will survive in the loop. This property holds for the major tones of the output signal. However, the proposed architecture induces other frequency spurs in addition to the two possible major frequencies. This is because the mixer in the feedback path always generates two frequencies at its output node. One produces the desired output frequency and the other results in the spurs. This spur circulates around the loop and creates another spur frequency. Therefore, the modified Miller divider can contain permanent spurs even if the circuits are ideal.

To illustrate these spur frequencies, two cases are considered in Fig. 4.

Case 1: $f_{\text {out }}=\left(f_{0}+\Delta f\right) / 2$

If the loop is settled, the frequency components at output node due to the two output frequencies of the feedback mixer can be written as

$$
\begin{aligned}
& f_{\text {out }}=f_{0}-\left(f_{\text {out }}-\Delta f\right)=\frac{1}{2} f_{0}+\frac{1}{2} \Delta f \\
& f_{\text {out }}^{\prime}=f_{0}-\left(f_{\text {out }}+\Delta f\right)=\frac{1}{2} f_{0}-\frac{3}{2} \Delta f .
\end{aligned}
$$

Equation (6) shows that the output frequency regenerates itself. However, (7) indicates an unexpected frequency spur is also generated. If the frequency in (7) feeds back through the mixers and the bandpass filter, similar to (6) and (7), the following equations can be obtained:

$$
\begin{aligned}
& f_{\text {out }}=f_{0}-\left(f_{\text {out }}^{\prime}+\Delta f\right)=\frac{1}{2} f_{0}+\frac{1}{2} \Delta f \\
& f_{\text {out }}^{\prime \prime}=f_{0}-\left(f_{\text {out }}^{\prime}-\Delta f\right)=\frac{1}{2} f_{0}+\frac{5}{2} \Delta f .
\end{aligned}
$$

The above equations show that the spur frequency in (7) contributes to the output frequency $f_{\text {out }}$ and results in one additional spur frequency $f_{\text {out }}^{\prime \prime}$ at the output node. The analysis can be performed recursively with the spur frequency obtained in (9) feeding back and other spur frequencies can be derived. However, (7) and (9) are the two major spurs because they are the nearest spur frequencies to the output frequency with maximum magnitude.

Case 2: $f_{\text {out }}=\left(f_{0}-\Delta f\right) / 2$

Similar analysis is performed, and the results are

$$
\begin{aligned}
& f_{\text {out }}=f_{0}-\left(f_{\text {out }}+\Delta f\right)=\frac{1}{2} f_{0}-\frac{1}{2} \Delta f \\
& f_{\text {out }}^{\prime}=f_{0}-\left(f_{\text {out }}-\Delta f\right)=\frac{1}{2} f_{0}+\frac{3}{2} \Delta f
\end{aligned}
$$

and

$$
\begin{aligned}
& f_{\text {out }}=f_{0}-\left(f_{\text {out }}^{\prime}-\Delta f\right)=\frac{1}{2} f_{0}-\frac{1}{2} \Delta f \\
& f_{\text {out }}^{\prime \prime}=f_{0}-\left(f_{\text {out }}^{\prime}+\Delta f\right)=\frac{1}{2} f_{0}-\frac{5}{2} \Delta f .
\end{aligned}
$$

Two spur frequencies are obtained. Both case 1 and case 2 show that two major spurs are far away from the main tone by $\pm 2 \Delta f$. In the MBOA-UWB mode-1 system, $2 \Delta f$ is approximately equal to $2 \mathrm{GHz}$. Therefore, the major spurs are out of band and can be filtered out at the output of downconversion mixer. Thus, in-band spurs become negligible for a modified Miller divider clock generator.

2) Settling Time Analysis: The transient response analysis of a regenerative frequency divider has been reported in [6]. In lieu of performing the analysis in time domain by differential equations [6], a simpler method, which can predict the settling behavior without complicated calculations, is employed to analyze the transient response of the modified Miller divider.

By transforming the proposed clock generator into frequency domain and building an equivalent model, the circuit becomes a linear system and can be analyzed in frequency domain. In Fig. 4, if both mixers in the modified Miller divider are ideal, the mixer can be modeled as an adder in the frequency domain because the main output frequency of the mixer is the sum or the difference of the two input signal frequencies, which means the nonlinear multiplier is simply a linear adder in frequency domain. Likewise, an ideal bandpass filter only adds a constant time delay to the signals in pass-band. Constant time delay is equivalent to linear phase shift with a constant scaling coefficient. In other words, the phase shift is equal to the product of the signal frequency and the constant delay time. Thus, applying the properties above, the modified Miller divider can be transformed into frequency domain shown in Fig. 7(a).

The upper feedforward path in Fig. 7(a) represents the additional phase shift introduced by the bandpass filter. Therefore, the transfer function $H(\omega)$ is equal to the constant delay time, $T_{\mathrm{D}}$, and is independent of signal frequency:

$$
H(\omega)=T_{D}
$$

A differentiator is cascaded with $H(\omega)$ to transform the phasedomain signal into a frequency- domain one. Therefore, the frequency-hopping behavior can be viewed as a frequency step applied to the input of the mixer on the feedback path. The magnitude of the step is equal to $+\Delta \omega$ or $-\Delta \omega$ depending on the output frequency after hopping is $\left(\omega_{0}-\Delta \omega\right) / 2$ or $\left(\omega_{0}+\Delta \omega\right) / 2$.

To simplify the analysis, two assumptions are made for the proposed model. First, like the PLL linear model, the amplitudes 


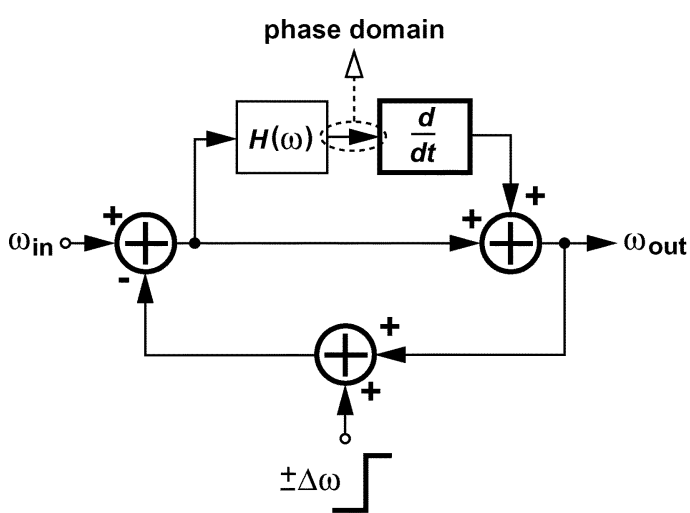

(a)

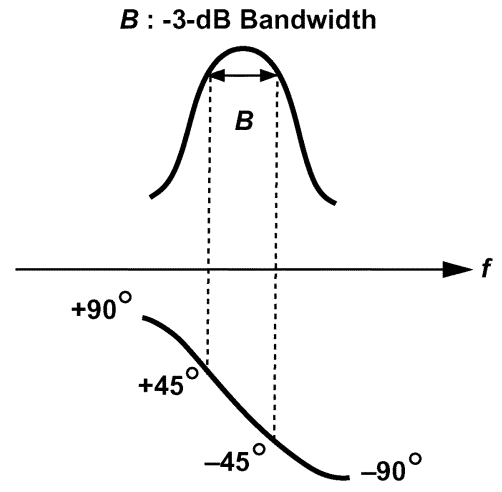

(b)

Fig. 7. (a) Linear model for a modified Miller divider in frequency domain. (b) Bode plot of a typical second-order bandpass filter.

of the node voltages have no effect on the settling behavior of the proposed clock generator as long as the amplitudes are sufficient to maintain proper operation. However, the amplitude gain of the bandpass filter does indeed affect the settling time. The loop gain must be chosen to be much greater than the minimum value to start oscillation, such that the settling time is dominated only by the bandwidth of the bandpass filter. Second assumption is that the output frequency should be within the pass-band of the bandpass filter. Due to the nonideality of the bandpass filter, the delay time is not a constant for all frequencies. With careful design, the output frequency can be centered to the pass-band frequency such that the delay time can be approximated by a constant during channel switching. In practice, both assumptions must be realized to ensure desired performance of the proposed clock generator.

The model in Fig. 7(a) becomes a linear system and the transfer function between $\omega_{\text {out }}$ and $\Delta \omega$ can be obtained by setting the input signal $\omega_{\text {in }}$ to zero

$$
\frac{\omega_{\mathrm{out}}}{\Delta \omega}=-\frac{s+\frac{1}{T_{D}}}{s+\frac{2}{T_{D}}} .
$$

Equation (15) shows that the modified Miller divider is a firstorder system in the frequency domain with a time constant $\tau$

$$
\tau=\frac{T_{D}}{2} .
$$

$T_{\mathrm{D}}$ is the constant delay time introduced by the bandpass filter and is simply equal to the slope in the phase diagram of the Bode plot. A typical second-order bandpass filter provides phase shift from $+45^{\circ}$ to $-45^{\circ}$ within the -3 -dB bandwidth as Fig. 7(b) shows.

We approximate the phase diagram by a straight line within the $-3-\mathrm{dB}$ bandwidth. Therefore, the delay time $T_{\mathrm{D}}$ is given as

$$
T_{D}=\frac{\pi / 2}{2 \pi B}=\frac{1}{4 B} .
$$

$B$ is the $3-\mathrm{dB}$ bandwidth. Then, (16) becomes

$$
\tau=\frac{T_{D}}{2}=\frac{1}{8 B}
$$

This model indicates that it takes about $7 \tau$ to settle to within $0.1 \%$ accuracy. Therefore, the channel-switching time is

$$
T_{\text {switching }} \approx 7 \tau=\frac{0.875}{B} \approx \frac{1}{B} \text {. }
$$

Equation (19) shows that the channel switching time can be approximated by the time constant corresponding to the bandwidth of the bandpass filter. As the previous conclusion shows, the quality factor $Q$ of the bandpass filter needs not to be high because the modified Miller divider adjusts itself to maintain a unique output frequency. Therefore, the $Q$ of the bandpass filter is chosen to be 5-7, i.e., the bandwidth is approximately equal to $500 \mathrm{MHz}$. Then, the estimated channel-switching time is about $2 \mathrm{~ns}$, much smaller than the UWB system requirement $9.5 \mathrm{~ns}$.

Fig. 8 shows the behavioral simulations of the modified Miller divider with different BPF bandwidths. The bandwidths are $50 \mathrm{MHz}, 100 \mathrm{MHz}, 250 \mathrm{MHz}$, and $500 \mathrm{MHz}$, and the corresponding channel switching times predicted by (19) are $20 \mathrm{~ns}, 10 \mathrm{~ns}, 4 \mathrm{~ns}$, and $2 \mathrm{~ns}$, respectively. These results confirm consistency between the prediction in (19) and circuit behavior.

\section{BUILDING BLOCKS}

Designed for the wireless receiver, the circuit needs to be low power. Furthermore, the number of passive inductors should be minimized to avoid large area. An active inductor technique with power optimization technique is proposed to achieve these two design goals simultaneously.

\section{A. Mixers}

Shown in Fig. 6, the clock generator consists of one feedforward mixer and one feedback mixer. Fig. 9 shows the doublebalanced mixer used in the feedback path with a resistive load $Z_{\mathrm{L}}$ due to lower frequency operation. The tail current source of the conventional double-balanced mixer is removed for lowvoltage and high-speed operation.

The feedforward mixer in Fig. 6 operates around $8 \mathrm{GHz}$, thus, $Z_{\mathrm{L}}$ can be replaced by an inductor for gain enhancement. However, the passive inductors required for the bandpass filter occupy significant silicon area. For example, a 3-nH inductor occupies area about $300 \mu \mathrm{m} \times 300 \mu \mathrm{m}$ in a $0.18-\mu \mathrm{m}$ CMOS tech- 


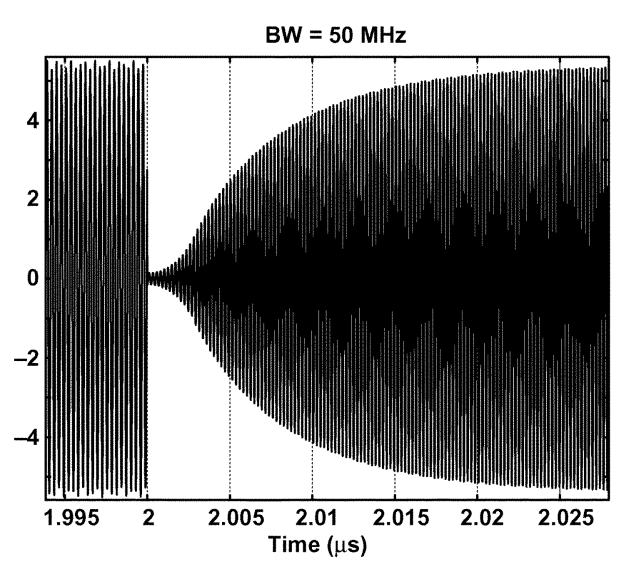

(a)

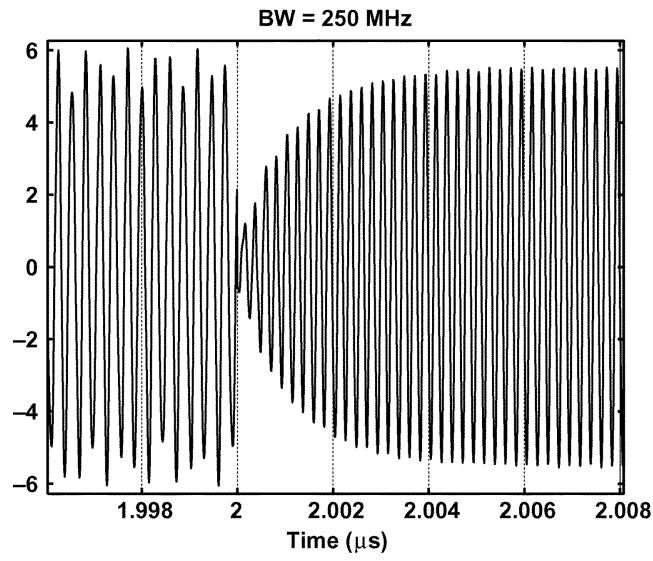

(c)

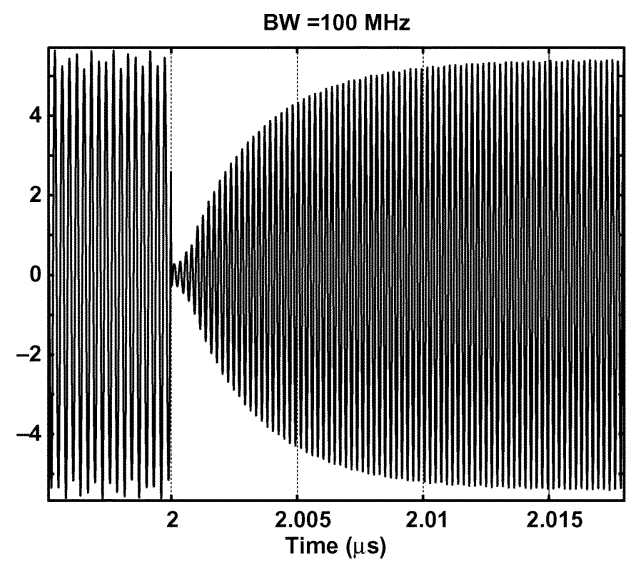

(b)

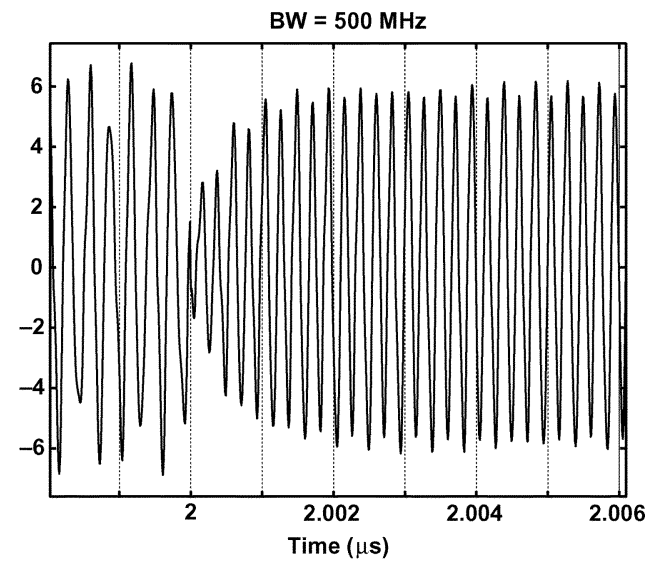

(d)

Fig. 8. Settling time simulation with different BPF bandwidth: (a) $50 \mathrm{MHz}$; (b) $100 \mathrm{MHz}$; (c) $250 \mathrm{MHz}$; (d) $500 \mathrm{MHz}$.

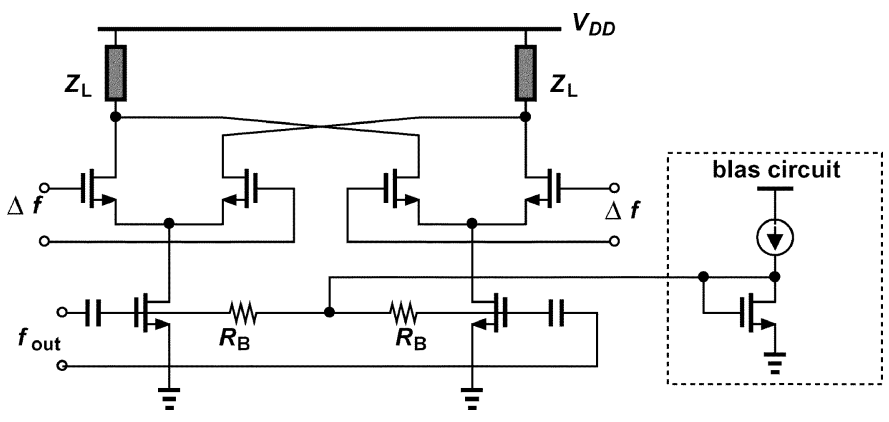

Fig. 9. Double-balanced mixer.

nology. Therefore, instead of using passive inductors, active inductors are widely used. For mobile application, the power consumption of RF active inductors must be minimized. In the next section, a technique for sizing the proposed active inductor is presented to minimize power dissipation.

\section{B. Active Inductor-Gyrator}

A gyrator with a capacitor $C$ can emulate an inductor in a parallel $L C$ filter [7]-[9]. Shown in Fig. 10, a gyrator is a $G_{m}$ cell with a negative $G_{m}$ cell on the feedback path. The effective impedance $Z_{\text {in }}$ can be derived as follows:

$$
L=\frac{C}{G_{m 1} G_{m 2}} .
$$

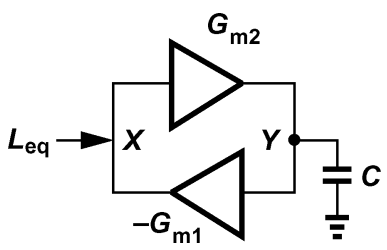

Fig. 10. Gyrator.

Equation (20) shows that the effective inductance of a gyrator is inverse proportional to the product of transconductance of the two $G_{m}$ cells and proportional to the capacitor capacitance $C$. In $\mathrm{GHz}$ applications, no extra capacitor but only the parasitic capacitance of the $G_{m}$ cell is at node $Y$. The effective inductance should be minimized to tune the center frequency to the desired value by maximizing each of the transconductance of the $G_{m}$ cells.

In CMOS technology, the transconductance can be increased either by using large aspect ratio devices at the cost of area or increasing the bias current at the cost of power consumption. Thus, for applications with stringent power consumption constraint, the transconductance can be increased only by using large devices, but large devices result in higher parasitic capacitance and lower resonant frequency. This design tradeoff complicates the active inductor design. 


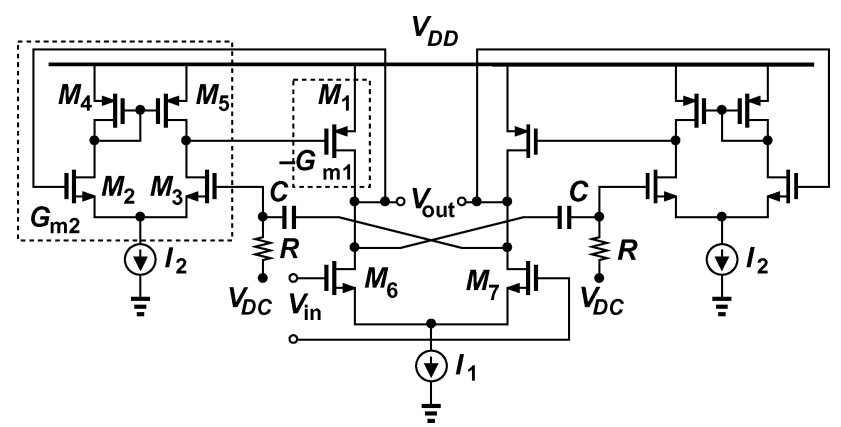

Fig. 11. Current-reusing tunable bandpass filter.

Fig. 11 shows the proposed active inductor design. The differential-to-single-ended amplifier represents the positive $G_{m}$ cell and the pMOS common-source amplifier forms the negative $G_{m}$ cell in the gyrator. Both $G_{m}$ cells have large output impedance. Therefore, without using additional $Q$-enhancement circuit [10], the overall $Q$ factor is large enough for this work. To enhance the effective transconductance of $G_{m 2}$, the resistor $R$ and capacitor $C$ form the AC coupling to increase the transconductance by a factor of two. Therefore, the effective transconductance $G_{m 2}$ seen at node $V_{\text {out }}+$ is approximately equal to $2 \mathrm{~g}_{m 2}$.

If $M_{2,3}$ and $M_{4,5}$ are perfectly matched

$$
L_{\text {ind }}=\frac{C_{p}}{G_{m 1} G_{m 2}}=\frac{C_{p}}{2 g_{m 1} g_{m 2}}
$$

where $C_{p}$ denotes the total parasitic capacitance at the drain of transistor $M_{3}$.

In order to make the resonant frequency higher, $g_{m 1}$ and $g_{m 2}$ can be larger by increasing the sizes of the transistors $M_{1}-M_{3}$, but at the cost of larger $C_{p}$. Thus, increasing the transconductances of the two amplifiers by increasing the width of the transistors arbitrarily cannot ensure higher resulting resonant frequency. To optimize the operation frequency of active inductors while consuming the minimum power, the following analysis is performed.

The capacitance $C_{p}$ consists of the parasitic capacitance of the transistors $M_{1}, M_{3}$, and $M_{5}$, and can be written as follows:

$$
C_{p}=C_{g s 1}+C_{d b 3}+C_{d b 5}+C_{g d 1}+C_{g d 3}+C_{g d 5} .
$$

Assuming all transistors operate in the saturation region, the gate-to-drain overlap capacitors are much smaller than the other parasitic capacitors and can be neglected. Thus, (22) becomes

$$
\begin{aligned}
C_{p} & \approx C_{g s 1}+C_{d b 3}+C_{d b 5} \\
& \approx \frac{2}{3} W_{1} L_{1} C_{\mathrm{ox}}+\gamma_{3} W_{3}+\gamma_{5} W_{5} \\
& =\frac{2}{3} W_{1} L_{1} C_{\mathrm{ox}}+\gamma_{2} W_{2}+\gamma_{5} W_{5} .
\end{aligned}
$$

Equation (23) also approximates $C_{\mathrm{db} 3}$ and $C_{\mathrm{db} 5}$ by their average values. Thus, the junction capacitance is simply proportional to the width of the transistor with a constant coefficient $\gamma$, determined by the process parameters.
From (21) and (23), the effective inductance becomes

$$
\begin{aligned}
L_{\text {ind }} & =\frac{C_{p}}{2 g_{m 1} g_{m 2}}=\frac{\frac{2}{3} W_{1} L_{1} C_{\mathrm{ox}}+\gamma_{2} W_{2}+\gamma_{5} W_{5}}{2 \sqrt{2 \mu_{p} C_{\mathrm{ox}} \frac{W_{1}}{L_{1}} I_{1}} \sqrt{2 \mu_{n} C_{\mathrm{ox}} \frac{W_{2}}{L_{2}} \frac{I_{2}}{2}}} \\
& =\frac{\frac{2}{3} W_{1} L_{1} C_{\mathrm{ox}}+\gamma_{2} W_{2}+\gamma_{5} W_{5}}{2 \sqrt{2 \mu_{p} \mu_{n}} C_{\mathrm{ox}} \times \sqrt{\frac{W_{1}}{L_{1}}} \sqrt{\frac{W_{2}}{L_{2}}} \sqrt{I_{1} I_{2}}} .
\end{aligned}
$$

The total capacitance $C_{\mathrm{x}}$ at node $V_{\text {out }}+$ can be estimated as

$$
\begin{aligned}
C_{x} & \approx C_{L}+C_{g s 2}+C_{d b 1} \\
& \approx C_{L}+\frac{2}{3} W_{2} L_{2} C_{\mathrm{ox}}+\gamma_{1} W_{1},
\end{aligned}
$$

where $C_{\mathrm{L}}$ represents the total load capacitance including the parasitic capacitance of $M_{6}$. However, (25) neglects gate-todrain overlap capacitance.

If all transistors have minimum channel length, $L_{\min }$, (26) can be obtained by multiplying (24) with (25):

$$
\begin{aligned}
& L_{\text {ind }} C_{x} \\
& =\frac{\left(\frac{2}{3} L_{\min } C_{\mathrm{ox}} W_{1}+\gamma_{2} W_{2}+\gamma_{5} W_{5}\right)\left(\gamma_{1} W_{1}+\frac{2}{3} L_{\min } C_{\mathrm{ox}} W_{2}+C_{L}\right)}{\left(\frac{2 C_{\mathrm{ox}}}{L_{\min }} \sqrt{2 \mu_{p} \mu_{n}} \sqrt{I_{1} I_{2}}\right) \sqrt{W_{1} W_{2}}} .
\end{aligned}
$$

The resonant frequency of a parallel $L C$ filter is the reciprocal of the square root of (26). Because transistor $M_{5}$ has no effect on the transconductance of either $G_{m}$ cell, minimum width of $M_{5}$ should be chosen to minimize the contribution of $C_{p}$ as long as $M_{5}$ operates in saturation region. Thus, if power consumption is limited (i.e., $I_{1}$ and $I_{2}$ both are fixed), only two parameters $W_{1}$ and $W_{2}$, the width of the transistors $M_{1}$ and $M_{2}$, can be varied. Take partial derivatives on (26) with respect to $W_{1}$ and $W_{2}$, respectively, and set both expressions equal to zero:

$$
\begin{aligned}
& \frac{\partial}{\partial W_{1}}\left(L_{\text {ind }} C_{x}\right)=0 \\
& \frac{\partial}{\partial W_{2}}\left(L_{\text {ind }} C_{x}\right)=0 .
\end{aligned}
$$

Divide one resulting equation by another and through algebraic simplification, (29) can be obtained:

$$
\begin{aligned}
& W_{1}\left(\frac{4}{3} L_{\min } C_{\mathrm{ox}} \gamma_{1} W_{1}+\frac{2}{3} L_{\min } C_{\mathrm{ox}} C_{L}+\gamma_{1} \gamma_{5} W_{5}\right) \\
& =W_{2}\left(\frac{4}{3} L_{\min } C_{\mathrm{ox}} \gamma_{2} W_{2}+\gamma_{2} C_{L}+\frac{2}{3} L_{\min } C_{\mathrm{ox}} \gamma_{5} W_{5}\right) .
\end{aligned}
$$

Equation (29) indicates that the parameters $W_{1}$ and $W_{2}$ must meet this constraint equation to minimize $L_{\mathrm{ind}} C_{\mathrm{x}}$ in (26). Typically, if $W_{1}, W_{2}$, and $W_{5}$ are small enough, the term containing load capacitance $C_{\mathrm{L}}$ dominates in the parenthesis of (29), therefore, (29) can be simplified to

$$
\begin{aligned}
\frac{2}{3} L_{\min } C_{\mathrm{ox}} C_{L} W_{1} & \approx \gamma_{2} C_{L} W_{2}, \\
\frac{W_{1}}{W_{2}} & =\frac{\gamma_{2}}{\frac{2}{3} L_{\min } C_{\mathrm{ox}}} .
\end{aligned}
$$


TABLE I

Process Parameters USED IN OPTIMAL Size CALCUlation

\begin{tabular}{|cc|}
\hline$L_{\min }$ & $0.18 \mu \mathrm{m}$ \\
$C_{\text {ox }}$ & $8.46 \mathrm{e}-3 \mathrm{~F} / \mathrm{m}^{2}$ \\
$\gamma_{2}$ & $0.769 \mathrm{nF} / \mathrm{m}$ \\
$\gamma_{5}$ & $0.900 \mathrm{nF} / \mathrm{m}$ \\
$W_{5}$ & $10 \mu \mathrm{m}$ \\
$C_{\mathrm{L}}$ & $0.2 \mathrm{pF}$ \\
\hline
\end{tabular}

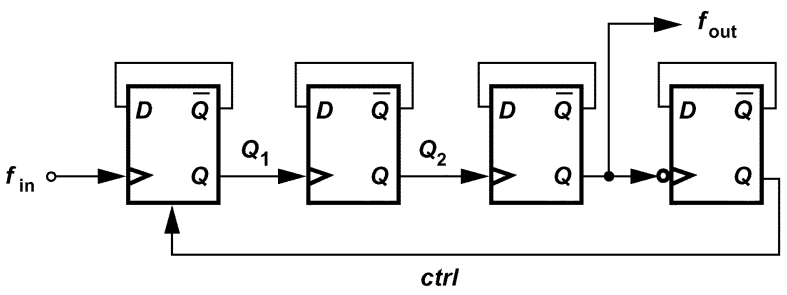

(a)

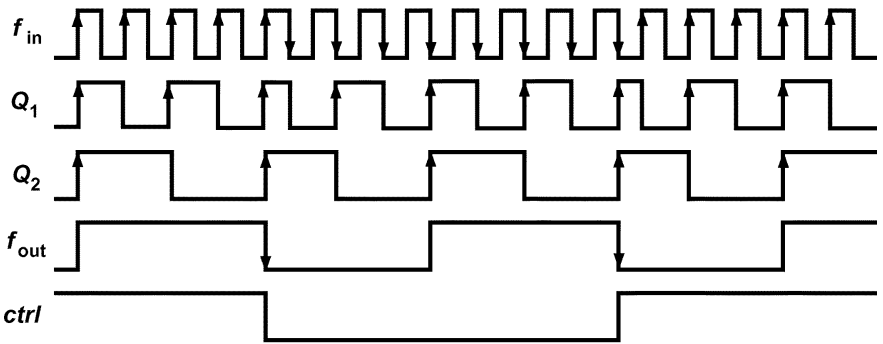

(b)

Fig. 12. (a) Divide-by-7.5 divider. (b) Timing diagram

Equation (30) shows that the width ratio between transistors $M_{1}$ and $M_{2}$ is proportional to a process-dependent constant, which is approximately equal to unity. With (29) and (30), the optimal size of $W_{1}$ can be expressed as

$$
W_{1} \approx \sqrt{\frac{3}{8} \frac{W_{5} C_{L} \gamma_{5}}{L_{\min } C_{\mathrm{ox}} \gamma_{2}}} .
$$

Combining with (30) and (31), the optimal sizes to maximize the resonant frequency can be found.

Table I summarizes the process parameters calculated from the TSMC $0.18-\mu \mathrm{m}$ CMOS process. The sizing of the tunable bandpass filter used in the modified Miller divider is designed according to (30) and (31) and the calculated values of $W_{1}$ and $W_{2}$ are $24 \mu \mathrm{m}$ and $32 \mu \mathrm{m}$, respectively.

\section{Divide-by-7.5 Divider}

Fig. 12 shows the circuit architecture of the divide-by-7.5 divider and the timing diagram. The control signal $c t r l$ selects the positive or negative edge to trigger the first D-flip-flop (DFF). The second and third DFFs are positive-edge trigger type while the fourth DFF is negative edge. All DFFs, except the first DFF, are used to implement the divide-by-2 function.

While the control signal $c t r l$ is positive, the circuit in Fig. 12 is an up-counting counter. However, when the control signal is negative, the output $\left(Q_{1}\right)$ of the first DFF changes state only after half of input reference period, providing the necessary 0.5

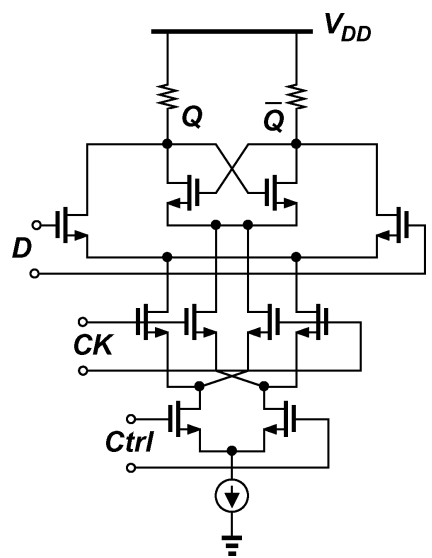

Fig. 13. Latch for edge-controlled DFF.

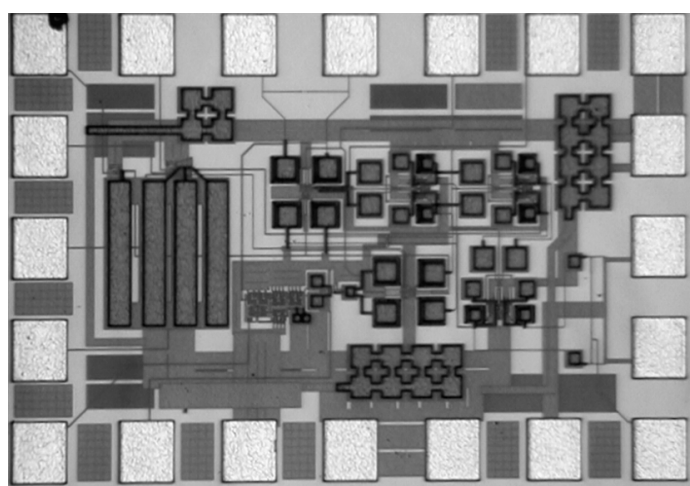

Fig. 14. Die photo.

cycle granularity to generate 7.5. The frequency of the output signal $f_{\text {out }}$ can thus be equal to $1 / 7.5$ of the input reference frequency.

Fig. 13 shows circuit implementation of the D latch in the edge-controlled DFF. The differential signal $c t r l$ steers the tail current to select the trigger signal for the first DFF. The rest of DFF's are conventional CML circuits.

\section{EXPERIMENTAL RESULTS}

The Miller-divider-based clock generator has been fabricated in a 0.18- $\mu \mathrm{m}$ CMOS technology. Shown in Fig. 14 is a photograph of the die, whose area is $860 \mu \mathrm{m} \times 600 \mu \mathrm{m}$. The circuit has been tested in a chip-on-board assembly while running from a 1.8-V power supply. The power dissipation is $47 \mathrm{~mW}$.

Fig. 15 shows the output spectrum for divide-by-2 operation only. The center frequency is $3960 \mathrm{MHz}$. The phase noise is equal to $-109.8 \mathrm{dBc} / \mathrm{Hz}$ at $100-\mathrm{kHz}$ offset.

Due to the process variation in the proposed clock generator, the settling time can be only measured in our another design, which only replaces all active inductors by equivalent passive inductors. Fig. 16 shows the transient response of the output by switching the frequency from $3960 \mathrm{MHz}$ to $4488 \mathrm{MHz}$. The measured settling time is less than $4 \mathrm{~ns}$, including the time to disable the direct feedback loop and then enable the loop with feedback mixer. Thus, the actual settling time of the proposed clock generator is about $2-3 \mathrm{~ns}$ with loop bandwidth around $500 \mathrm{MHz}$, consistent with the prediction of the proposed linear model. In 


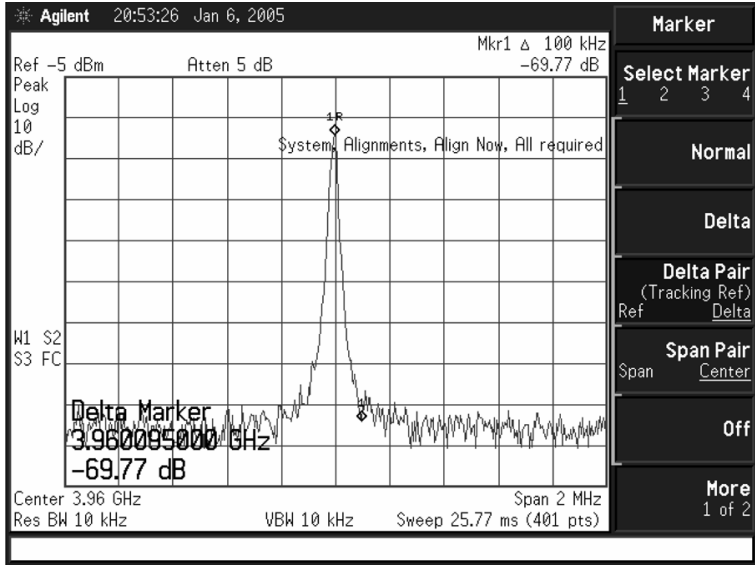

Fig. 15. Output spectrum at $3960 \mathrm{MHz}$.

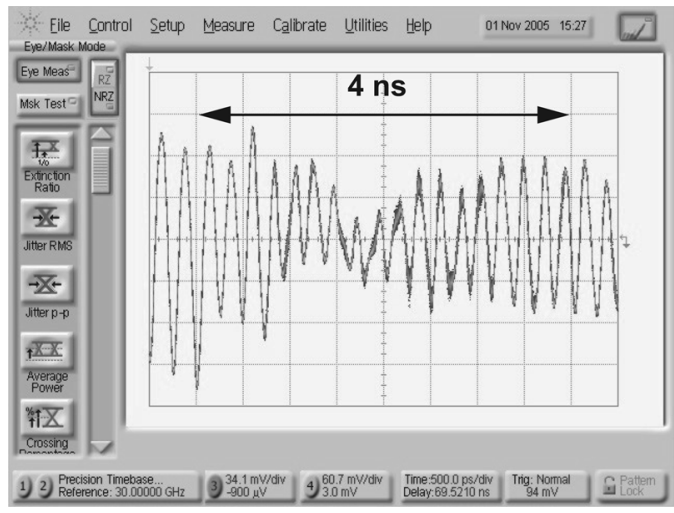

Fig. 16. Settling behavior of switching output frequency from $3960 \mathrm{MHz}$ to $4488 \mathrm{MHz}$.

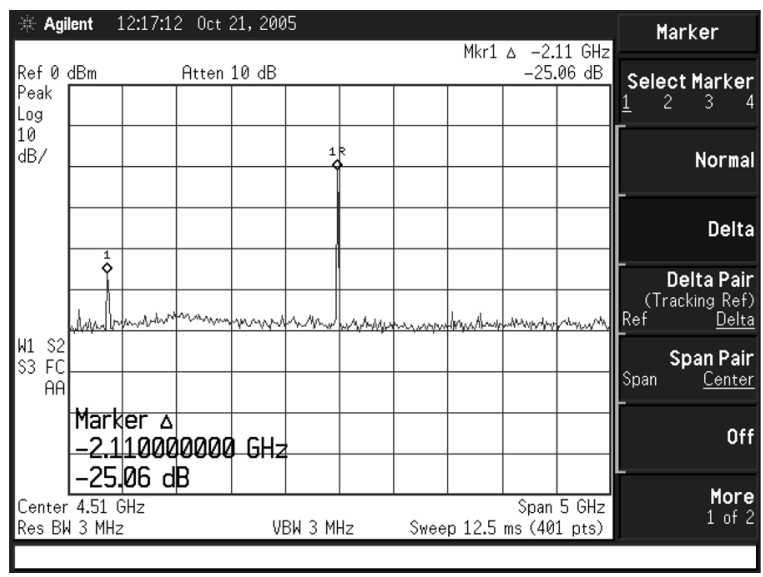

Fig. 17. Output spectrum at $4488 \mathrm{MHz}$.

Fig. 17, the output spectrum at $4488 \mathrm{MHz}$ clearly shows that the circuit maintains only one signal frequency at $4488 \mathrm{MHz}$ and suppresses the 3432-MHz component. The major spur frequency is about $2 \mathrm{GHz}(2 \Delta f)$ away from the main tone which meets the spur analysis prediction. Table II summarizes the performance of this clock generator.
TABLE II

PERFORMANCE SUMMARY

\begin{tabular}{|lc|}
\hline Carrier Frequency & $3432 \mathrm{MHz}$ \\
& $3960 \mathrm{MHz}$ \\
& $4488 \mathrm{MHz}$ \\
Phase noise at 100-kHz offset & $-109.8 \mathrm{dBc} / \mathrm{Hz}$ \\
SettlingTime (simulation) & $<9.5 \mathrm{~ns}$ \\
Power Dissipation & $47 \mathrm{~mW}$ \\
Supply Voltage & $1.8 \mathrm{~V}$ \\
Die Area & $0.86 \mathrm{~mm} \times 0.6 \mathrm{~mm}$ \\
Technology & $0.18-\mu \mathrm{m} \mathrm{CMOS}$ \\
\hline
\end{tabular}

\section{CONCLUSION}

A Miller-divider-based clock generator is proposed to generate the three carrier frequencies of the MBOA-UWB mode-1 system while achieving less than 9.5-ns frequency settling time. The proposed approach adds a feedback mixer in the conventional Miller divider circuit and the desired output frequency is determined by the bandpass filter. A linear model is also introduced to estimate the frequency-switching time by transforming the circuit into frequency domain. To save chip area, active inductors are used in the circuit design and an optimization technique is also presented to minimize power consumption of the proposed active inductor. The proposed concepts are demonstrated in a $0.18-\mu \mathrm{m}$ CMOS technology.

\section{ACKNOWLEDGMENT}

The authors would like to thank Mediatek and the National Science Council for chip fabrication.

\section{REFERENCES}

[1] C. C. Lin and C. K. Wang, "Subharmonic direct frequency synthesizer for mode-1 MB-OFDM UWB system," in Symp. VLSI Circuits Dig. Papers, Jun. 2005, pp. 38-41.

[2] C. C. Lin and C. K. Wang, "A regenerative semi-dynamic frequency divider for mode-1 MB-OFDM UWB hopping carrier generation," in IEEE ISSCC Dig. Tech. Papers, Feb. 2005, pp. 206-207.

[3] T. C. Lee and Y. C. Huang, "A miller divider based clock generator for MBOA-UWB application," in Symp. VLSI Circuits Dig. Papers, Jun. 2005, pp. 34-37.

[4] R. L. Miller, "Fractional-frequency generators utilizing regenerative modulation," Proc. Inst. Radio Eng, vol. 27, pp. 446-456, Jul. 1939.

[5] J. Lee and B. Razavi, "A 40-GHz frequency divider in 0.18- $\mu \mathrm{m}$ CMOS technology," IEEE J. Solid-State Circuits, vol. 39, no. 4, pp. 594-601, Apr. 2004.

[6] C. W. Helstrom, "Transient analysis of regenerative frequency dividers," IEEE Trans. Circuit Theory, pp. 489-497, Dec. 1965.

[7] Y. T. Wang and A. A. Abidi, "CMOS active filter design at very high frequencies," IEEE J. Solid-State Circuits, vol. 25, no. 6, pp. 1562-1574, Dec. 1990.

[8] A. Thanachayanont and A. Payne, "VHF CMOS integrated active inductor," Electron. Lett., vol. 32, no. 11, pp. 999-1000, May 1996.

[9] M. Grözing, A. Pasht, and M. Berroth, "A 2.5 V CMOS differential active inductor with tunable $\mathrm{L}$ and $\mathrm{Q}$ for frequencies UP to $5 \mathrm{GHz}$," in Proc. IEEE Radio Frequency Integrated Circuit (RFIC) Symp., May 2001, pp. 575-578.

[10] U. Yodprasit and J. Ngarmnil, "Q-enhancing technique for RF CMOS active inductor," in Proc. IEEE Int. Symp. Circuits and Systems, May 2000, pp. 589-592. 


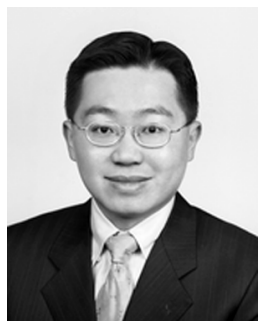

Tai-Cheng Lee (S'91-M'95) was born in Taiwan, R.O.C., in 1970. He received the B.S. degree from National Taiwan University, Taipei, Taiwan, in 1992, the M.S. degree from Stanford University, Stanford, CA, in 1994, and the Ph.D. degree from the University of California, Los Angeles, in 2001, all in electrical engineering.

He worked for LSI Logic from 1994 to 1997 as a Circuit Design Engineer. He served as an Adjunct Assistant professor at the Graduate Institute of Electronics Engineering (GIEE), National Taiwan University, from 2001 to 2002. Since 2002, he has been with the Electrical Engineering Department and GIEE, National Taiwan University, where he is an Assistant Professor. His research interests include data converters, PLL systems, and RF circuits.

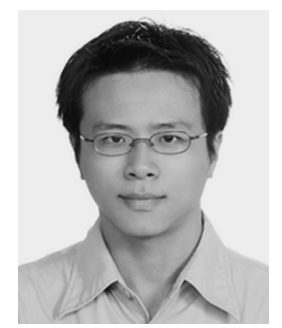

Yen-Chuan Huang was born in Taiwan, R.O.C., in 1980. He received the B.S. and M.S. degrees in electrical engineering from National Taiwan University, Taipei, Taiwan, in 2003 and 2005, respectively. He is currently working toward the Ph.D. degree in electronics engineering at National Taiwan University. 